\title{
It is time to individualize anti-CD20 therapies in multiple sclerosis
}

\author{
Jagannadha Avasarala ${ }^{1}$ \\ ${ }^{1}$ University of Kentucky Medical Center
}

March 23, 2021

\begin{abstract}
Anti-CD20 therapies in multiple sclerosis (MS) have become central to management of the disease since their FDA approval in 2017. As their role in MS management continues to grow, it is also increasingly important to know how such drugs can be better administered using current knowledge of how B cells repopulate after their depletion. To this end, individualizing therapy needs to be prioritized since a timed-dosing interval is perhaps not required based on evidence and it certainly unwelcome from a financial perspective.
\end{abstract}

It is time to individualize anti-CD20 therapies in multiple sclerosis

Jagannadha Avasarala, MD, PhD

Department of Neurology, University of Kentucky Medical Center and Kentucky Neuroscience Institute, Lexington, KY 40536, USA

Correspondence:

Jagannadha Avasarala, MD, PhD

Professor of Neurology

Director, Comprehensive MS Center

Department of Neurology

University of Kentucky Medical Center

Kentucky Neuroscience Institute

740 S Limestone

Lexington, KY 40536

WC: 1365

The first and only self-administered CD20 depleting therapy for relapsing forms of multiple sclerosis (MS), ofatumumab (Kesimpta), was approved by the FDA in August 2020. It is an immunoglobulin class G1 kappa monoclonal antibody (Mab) that selectively binds to an epitope on the cell surface protein, CD20. The CD20 is a transmembrane cellular protein that is a therapeutic target in B-cell malignancies and is expressed in over $95 \%$ of B-cell lymphocytes throughout their development but is absent in hematopoietic stem cells. In the IV administered form, Arzerra/ofatumumab, FDA-approved for use in patients with previously untreated chronic lymphocytic leukemia (CLL) in October, 2009, B-cell depletion in the peripheral blood was persistent after six months following the last dose. In vitro, ofatumumab induces complement-dependent cytotoxicity (CDC) and antibody-dependent cellular cytotoxicity (ADCC) which contribute to depletion of CD20 cells in peripheral blood. 
According to the package insert, the recommended dosing regimen for Kesimpta/ofatumumab in RMS is a monthly dose of $20 \mathrm{mg}$, given subcutaneously, after an initial dosing regimen protocol. Thepackage insert for any drug is a document that was first developed in 1966, amended in 2006 and carries potential legal weight; it also includes data supplied by the manufacturer that is evaluated/approved by the Food and Drug Administration (FDA). In clinical trials leading up to the approval of the drug, approximately 1500 patients with RMS received ofatumumab. In study 1 (NCT02792218) and study 2 (NCT02792231), 1882 patients with RMS were randomized, 946 of whom were treated with ofatumumab for a median duration of 85 weeks; about $33 \%$ of patients receiving ofatumumab were treated for up to 120 weeks. In both studies, ofatumumab administration resulted in a reduction of CD19+ B-cells to below the LLN in $77.0 \%$ and $78.8 \%$ of patients, respectively, one week after treatment initiation, and in $95.0 \%$ and $95.8 \%$ of patients, respectively, two weeks after treatment initiation. To follow how B-cell depletion occurs, assays for CD19+ B-cells were used because the presence of the drug interferes with the CD20 assay.

In both studies, at week 12, 99.3\% to $99.5 \%$ of patients had CD19+ B-cell counts below LLN. The CD19+ B-cell counts remained below LLN for approximately $97 \%$ of patients in Study 1 and $92 \%$ of patients in Study 2 from 12 weeks through 120 weeks while on treatment. Modeling and simulation for B-cell repletion corroborates these data, predicting median time to B-cell recovery of 40 weeks post-treatment discontinuation.

Comparative pharmacokinetic data for Arzerra/ofatumumab shows that owing to depletion of B cells that follows each subsequent infusion, drug clearance is significantly decreased as compared to its clearance after the first infusion of the drug. This is attributed to its clearance by both a target-independent and a B-cell mediated route. The initial dosing for Arzerra is $300 \mathrm{mg} / \mathrm{kg}$ followed by seven weekly and four monthly infusions of $2000 \mathrm{mg} / \mathrm{kg}$ per dose. The $\mathrm{C}_{\max }$ were $40 \%$ higher and area under curve (AUC) were $60 \%$ higher after the $8^{\text {th }}$ infusion than after the $4^{\text {th }}$ infusion. This points to decreased drug clearance when $\mathrm{B}$ cell populations are depleted. One of the most consistent findings in animal and human studies is the variability of depletion seen with anti-CD 20 MAbs in different individuals even when treated with the same dose ${ }^{1}$. Interestingly, depletion in the same individual tends to be consistent in different tissues, suggesting that individual characteristics are probably important.

For Kesimpta/ofatumumab, a study of bioequivalence before initiation of the maintenance phase, total CD19+ B-cell levels below the defined threshold of 10 cells $/ \mu \mathrm{L}$ were achieved in $94 \%$ of patients starting at week 4 and in $98 \%$ of patients at week 12. B-cell repletion data from RMS clinical studies indicate B-cell recoveries over the LLN in about $50 \%$ of patients in 24-36 weeks post-treatment discontinuation. In a study that provided class I evidence, the MIRROR study ${ }^{2}$, the authors noted a dose-dependent depletion of B cells, (Figure 1) with the most effect for the $60 \mathrm{mg}$ dose administered every 12 weeks (the recommended dose is $20 \mathrm{mg}$ q weekly, per the package insert for Kesimpta) and in this cohort, the B cell reconstitution ranged from 85-777 days (323.4 days, mean). The effect of cumulative or subsequent dosing on B cell depletion was not studied in the MIRROR study. Individualized treatment schedules need to be developed given the wide range of $\mathrm{B}$ cell repopulation kinetics, i.e., 85-777 days.

Reconstitution of B cells is complex and cells that repopulate have a different immunological profile. The first wave of repopulating B cells are immature B cells $\left(\mathrm{CD} 38^{\text {high }}, \operatorname{IgD}+, \mathrm{CD} 10+, \mathrm{CD} 24^{\text {high }}\right)$, and the immunoglobulin receptors are not yet somatically mutated ${ }^{3}$. Additionally, the number of naive B cells increase, and they predominate in the peripheral blood B cell pool. CD27+ memory B cells show a slow and delayed repopulation, but their numbers stay significantly reduced $(<50 \%)$ compared to baseline ${ }^{3}$. Repopulation occurs mainly with naïve B cells and transitional B cells similar to that seen after bone marrow transplantation ${ }^{4}$. In a recent publication ${ }^{5}$, repopulating B cells after anti-CD20 therapy were shown to display a phenotype that is transitional and naïve, with a reduction in memory B cells. Reappearing B cells showed activation of CD25, CD69, and expressed higher levels of costimulatory CD40 and CD86 markers. Most importantly, B-cell repopulation kinetics display both high inter-and intra-individual variance and a higher body mass index (BMI) may be associated with faster repopulation rates ${ }^{6}$. One course of treatment with Rituxan, the original CD20 depleting drug, was followed by B-cell repopulation of the peripheral blood 
starting usually within 6-9 months - but it can take several months or even years for total B-cell numbers recover to pretreatment levels. Hence, re-dosing that is not based on B cell kinetics, either qualitative or quantitative, or both, is not based on evidence.

In studies that have investigated the depletion of CD20-expressing B cells in solid tissues, it has been shown that depletion is significant but not complete, with bone marrow and spleen being more easily depleted than lymph nodes ${ }^{7}$. Depletion of B cells is thought to include not only the total drug dose administered and distribution into various tissues, but also B-cell intrinsic and microenvironment factors influencing recruitment of effector mechanisms and antigen and effector modulation. The degree of $\mathrm{B}$ cell depletion varies between individuals even for the same dosage but does not have significant variation in the same individual suggesting that individual factors are important in determining the final extent of depletion, a finding ideal for designing individualized therapy in MS. Furthermore, CD20 depletion is associated with immunoglobulin - IgM and then IgA and IgG hypogammaglobulinaemia in some cases, predisposing patients to a small but increased risk of severe infections ${ }^{8}$.

It is unclear why Kesimpta/ofatumumab needs to be dosed monthly since there are no data to support that rationale. Most importantly, no formal review of Kesimpta/ofatumumab for MS is available through the Center for Drug Evaluation and Research (CDER) and the FDA does not have pharmacokinetic (PK) data published on its website for this product. Absent a formal review by CDER, dosing schedules for Kesimpta/ofatumumab are probably extrapolated from a combination of assessment of ofatumumab (Arzerra) by CDER and the MIRROR study findings - a clear rationale is not provided. Data for Arzerra/ofatumumab is available from that product's review in the past, however. Although the depletion of B-cells in the peripheral blood had a measurable pharmacodynamic effect for Arzerra/ofatumumab, it was not directly correlated with the depletion of B cells in solid organs and the effect of B-cell depletion was not linked to clinical response (package insert, Arzerra). If clinical responsiveness is not related to B-cell depletion in CLL, how sure are we that the depletion of CD20 cells in MS secondary to Kesimpta/ofatumumab, drives the clinical response?

In conclusion, an individualized dosing approach to anti-CD20 therapies is long overdue because 1) repopulation of B cells shows high intra and inter-individual variance, 2) naïve B cells are the predominant cell type in repopulated cells 3) B cell depletion may be dependent, in part, on BMI, 4) the clearance of Arzerra/ofatumumab requires the existence of B cell populations with subsequent dosing(s) and 5) increased risk of infections associated with hypogammaglobulinemia observed in patients using 6 monthly dosing of anti-CD20 drugs. Why then are dosing schedules for anti-CD20 drugs not personalized ?

Conflict of interest: None

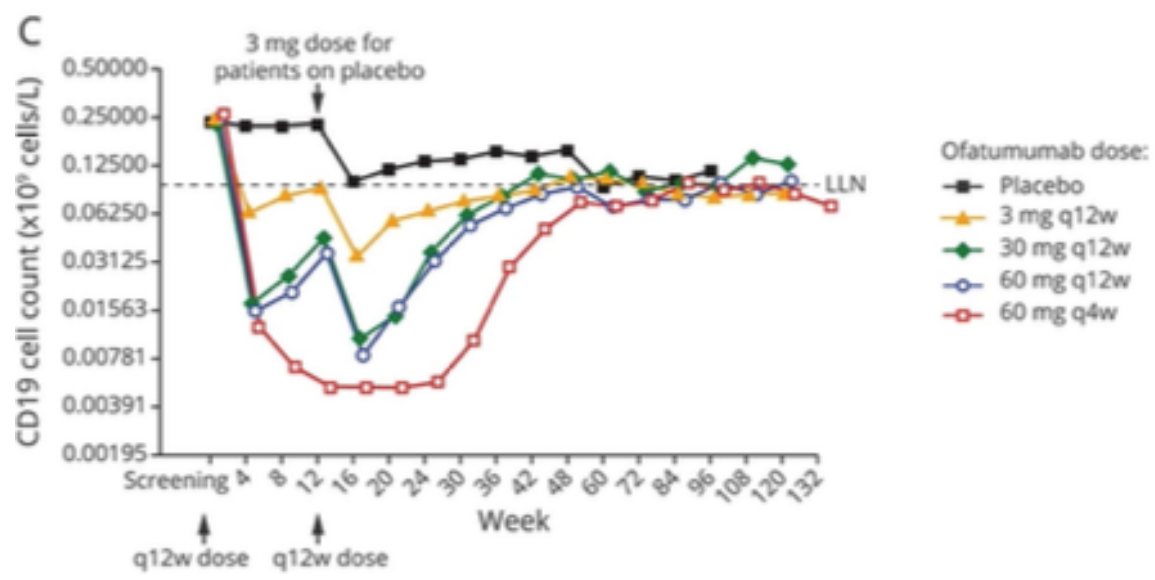

Figure 1 : From the MIRROR study, showing the effect of Ofatumumab dosing strength(s) and intervals on CD19 population metrics. 
LLN is lower limit of normal.

\section{References}

1. Reff ME, Carner K, Chambers KS, et al. Depletion of B cells in vivo by a chimeric mouse human monoclonal antibody to CD20. Blood 1994; 83: 435-445. 2. Bar-Or A, Grove RA, Austin DJ, et al. Subcutaneous ofatumumab in patients with relapsing-remitting multiple sclerosis: The MIRROR study. Neurology 2018; 90: e1805-e1814. 3. Roll P, Palanichamy A, Kneitz C, Dorner T, Tony HP. Regeneration of B cell subsets after transient B cell depletion using anti-CD20 antibodies in rheumatoid arthritis. Arthritis Rheum 2006; 54: 2377-2386. 4. Bemark M, Holmqvist J, Abrahamsson J, Mellgren K. Translational MiniReview Series on B cell subsets in disease. Reconstitution after haematopoietic stem cell transplantation revelation of B cell developmental pathways and lineage phenotypes. Clin Exp Immunol 2012; 167: 15-25. 5. Nissimov N, Hajiyeva Z, Torke S, et al. B cells reappear less mature and more activated after their anti-CD20-mediated depletion in multiple sclerosis. Proc Natl Acad Sci U S A. 2020; 117: 25690-25699. 6. Signoriello E, Bonavita S, Di Pietro A, et al. BMI influences CD20 kinetics in multiple sclerosis patients treated with ocrelizumab. Mult Scler Relat Disord 2020); 43: 102186. 7. Leandro MJ. B-cell subpopulations in humans and their differential susceptibility to depletion with anti-CD20 monoclonal antibodies. Arthritis Res Ther 2013; 15 (Suppl 1): S3. 8. Baker D, Roberts CAK, Pryce G, et al. Covid 19 vaccine readiness for anti-CD20-depleting therapy in autoimmune diseases. Clin Exp Immunol 2020; 202: 149-161. 